\title{
Complicaciones agudas en adultos con diagnóstico de diabetes mellitus tipo 1 y 2 ingresados al servicio de emergencia de un hospital de tercer nivel
}

\section{Acute complications in adults diagnosed with type 1 and 2 diabetes mellitus admitted to the emergency department of a tertiary level hospital}

\author{
Omar Fabricio Zanoni Ramos', Katherine \\ Leslie Marin Pimentel, Karen Gabriela Luyo \\ Fajardo, Carolina Sarria Arenaza², Guiliana \\ Mas Ubillús ${ }^{3}$
}

Zanoni-Ramos O, Marin-Pimentel K, Luyo-Fajardo K, Sarria-Arenaza C, Mas-Ubillús $\mathrm{G}$. Complicaciones agudas en adultos con diagnóstico de diabetes mellitus tipo I y 2 ingresados al servicio de emergencia de un hospital de tercer nivel. Rev Soc Peru Med Interna. 2021;34(4):196-200. DOI: https://doi.org/10.36393/spmi.v34i4.638

\section{RESUMEN}

Objetivo: Describir las características de las complicaciones agudas de la diabetes en los pacientes que acuden al servicio de emergencia de un hospital general de tercer nivel. Material y métodos: Estudio descriptivo y prospectivo en pacientes que acudieron al servicio de Emergencia del Hospital Nacional Arzobispo Loayza (HNAL), Lima, entre septiembre 2019 y marzo 2020, con complicaciones agudas de la diabetes mellitus. Se registraron las características demográficas, clínicas, laboratoriales y destino del paciente. Los datos fueron analizados con el programa STATA 14. Resultados: Ingresaron a la emergencia 22 pacientes con alguna complicación aguda de la DM. Quince pacientes tenían crisis hiperglucémica y siete con hipoglicemia. De los pacientes con crisis hiperglicémica, I 2 (80\%) fueron cetoacidosis y 3 (20\%) estado hiperosmolar. El 46,7\% presentó diabetes de diagnóstico reciente. El factor desencadenante más frecuente fue las infecciones en el $60 \%$. El $50 \%$ de pacientes con cetoacidosis tuvo presentación severa. Conclusión: La cetoacidosis es la complicación aguda más frecuente en pacientes con diabetes mellitus tipo 2 y el desencadenante más frecuente fueron las infecciones.

Palabras clave: diabetes mellitus, hiperglicemia, hipoglicemia, cetoacidosis, estado hiperosmolar.

Médico cirujano. Facultad de Medicinas, Universidad Peruana Cayetano Heredia, Lima, Perú.

Médica endocrinóloga. Servicio de Endocrinología, Hospital

Nacional Arzobispo Loayza. Facultad de Medicina, Universidad Peruana Cayetano Heredia, Lima, Perú

3 Médica internista. Servicio de Emergencia. Hospital Nacional Arzobispo Loayza. Facultad de Medicina, Universidad Peruana Cayetano Heredia, Lima, Perú.

ORCID ID: https://orcid.org/0000-0003-037I-209X 


\begin{abstract}
Objective:To describe the characteristics of acute complications of diabetes in patients attending the emergency service of a thirdlevel general hospital. Material and methods: Descriptive and prospective study in patients who attended the Emergency Service of the Arzobispo Loayza National Hospital (HNAL), Lima, between September 2019 and March 2020, with acute complications of diabetes mellitus. The demographic, clinical, laboratory and destination characteristics of the patient were recorded. Data were analyzed with the STATA 14 program. Results: 22 patients with some acute complications of DM were admitted to the emergency room. Fifteen patients had hyperglycemic crises and seven had hypoglycemia. Of the patients with hyperglycemic crises, 12 (80\%) were ketoacidosis and 3 (20\%) were hyperosmolar. 46,7\% had newly diagnosed diabetes. The most frequent trigger was infections in $60 \% .50 \%$ of patients with ketoacidosis had severe presentation.
\end{abstract}

Conclusion: Ketoacidosis is the most frequent acute complication in patients with type 2 diabetes mellitus type 2 and the most frequent trigger was infections.

Keywords: diabetes Mellitus, hyperglycemia, hypoglycemia, ketoacidosis, hyperosmolar state.

\section{INTRODUCCIÓN}

La diabetes mellitus (DM) es un desorden metabólico crónico, multicausal, originado por una secreción inadecuada de insulina o defecto en la acción de la misma, que altera la metabolización de macronutrientes. Puede clasificarse en DM tipo 1 (DM1), que representa el 5-10\% de los casos, y DM tipo 2 (DM2) que constituye el 90-95\%. Esta patología puede generar complicaciones agudas que pueden ser precipitadas por la deshidratación, transgresión alimentaria y efectos farmacológicos ${ }^{1} ; \mathrm{y}$, complicaciones a largo plazo a nivel microvascular y macrovascular.

A nivel mundial, se ha estimado que 463 millones de pacientes, entre 20 a 79 años, padecían DM en el año 2019, y se espera que para el 2030 esta cifra aumente a 578 millones. ${ }^{2}$ En nuestro país, la Federación Internacional de Diabetes (IDF) indicó que 1385000 peruanos, entre 20 y 79 años, padecían DM en ese mismo año. ${ }^{2}$ Esta condición acarrea un alto gasto económico a largo plazo, por lo que constituye un problema de salud pública de creciente importancia en nuestro país.

Las complicaciones agudas de la diabetes son estados de descompensación que aumentan la morbimortalidad en los diabéticos. Dichas complicaciones pueden ser crisis hiperglucémicas $(\mathrm{CH})$, que incluyen la cetoacidosis diabética (CAD), estado hiperosmolar (EH) y estado mixto (EM), o pueden ser no hiperglucémicas, como la hipoglucemia. ${ }^{3}$

En el Perú, diversos estudios han descrito las complicaciones causadas por la DM en pacientes de diferentes hospitales del país. Un estudio prospectivo de 110 pacientes con $\mathrm{CH}$, durante el periodo 2001-2002, demostró que el 49,1\% correspondió a CAD, 8\% a EH y $46 \%$ a EM. ${ }^{4}$ Asimismo, otro estudio reportó que la CAD fue la complicación aguda más común en hospitalización. ${ }^{5}$ Por otro lado, otra serie de pacientes con DM2 y CAD, observó que el $66 \%$ tuvo la forma severa, y los principales factores desencadenantes fueron la discontinuación del tratamiento (40\%) e infecciones $(30 \%){ }^{6}$ Finalmente, respecto a las hipoglucemias, sólo un estudio reportó que fue la complicación aguda con la tasa más alta de hospitalización (10,6\%) en pacientes con DM2. ${ }^{7}$ Dado el impacto de la DM y sus complicaciones, es importante desarrollar estudios actualizados para describir el panorama de las complicaciones agudas de la diabetes en nuestro medio. Por ello, el objetivo del presente estudio fue describir las características epidemiológicas, clínicas y laboratoriales de las principales complicaciones agudas (CAD, EH, EM e hipoglucemia) en los pacientes con diagnóstico o reciente diagnóstico de DM1 y DM2.

\section{MATERIAL Y MÉTODOS}

Diseño del estudio: se realizó un estudio descriptivo y prospectivo tipo serie de casos desde septiembre del 2019 a marzo del 2020.

Participantes: se incluyó a los pacientes diabéticos o con reciente diagnóstico de diabetes que acudieron al Servicio de Emergencia del Hospital Nacional Arzobispo Loayza (HNAL), Lima, con el diagnóstico de alguna complicación aguda de la DM (hipoglucemia, cetoacidosis diabética, estado hiperosmolar hiperglucémico o estado mixto). En el caso de hipoglucemia, debió tener el antecedente de DM1 o DM2. Las pacientes gestantes fueron excluidas de este estudio.

Recolección de datos: se recolectaron datos sociodemográficos y clínicos de las historias clínicas de los pacientes mediante una ficha de recolección de datos, desde el 23 de septiembre del 2019 hasta el 14 de marzo del 2020. Previo a la recolección de los datos, se solicitó el consentimiento informado de los pacientes para la revisión de su historia clínica. Los datos fueron digitados en una hoja de cálculo de Microsoft Excel 2016.

Análisis estadístico: para las variables cuantitativas se calculó la mediana y el rango intercuartil. Para las variables cualitativas se calculó los porcentajes y frecuencias. Los datos fueron ingresados en el programa de STATA 14.

Aspectos éticos: el proyecto de investigación se llevó a cabo con previa autorización del Comité de Ética de la Universidad Peruana Cayetano Heredia y de la Oficina de Docencia del HNAL.

\section{RESULTADOS}

Se incluyó un total de 22 pacientes, 15 con crisis hiperglucémicas y 7 con hipoglucemia. Los pacientes 
$(13,33 \%)$ DM1. Se encontró que todos los pacientes con DM1 y con DM2 presentaron CAD (ver Tabla 1), mientras que los pacientes con diabetes de reciente diagnóstico, 4 $(57,14 \%)$ presentaron CAD y $3(42,85 \%) \mathrm{EH}$. Respecto a los factores desencadenantes, en $9(60 \%)$ pacientes fue un proceso infeccioso, en $2(13,33 \%)$ fue la falta de tratamiento y en 1 pancreatitis aguda $(6,67 \%)$. En la Tabla 1 también se muestran las características clínicas de los pacientes con crisis hiperglucémicas. Luego, en base a los resultados de los exámenes de laboratorio (Tabla 2) se determinó la severidad de la CAD, mostrando que $6(50 \%)$ presentaron un grado severo, $5(41,67 \%)$ moderado y $1(8,33 \%)$ leve. De los pacientes que presentaron hipoglucemia, la mayoría fueron varones $(85,71 \%, n=6)$ y la mediana de la edad y tiempo de enfermedad fue 66 años y 6 horas, respectivamente. La mediana de glucosa sérica fue 35 $\mathrm{mg} / \mathrm{dl}$. Seis pacientes $(85,71 \%)$ ingresaron al servicio de emergencia de medicina, mientras que uno (14,29\%) ingresó por la Unidad de Shock Trauma, siendo este último el único fallecido en esta serie. El factor desencadenante más frecuente fue la no ingesta de alimentos $(71,43 \%)$. Se encontró que 5 pacientes utilizaron glibenclamida, 4 metformina y 1 utilizaba insulina. En la Tabla 3 se describen las características clínicas y laboratoriales de este grupo de pacientes.

\section{DISCUSIÓN}

Se reclutaron 22 pacientes con alguna complicación aguda de la DM. La complicación más frecuente fue la cetoacidosis diabética (CAD), seguida de la hipoglucemia y finalmente el estado hiperosmolar. Estos resultados concuerdan con otros realizados en nuestro medio, donde la CAD fue la complicación aguda más frecuente. ${ }^{4,8}$ Esto puede atribuirse a que la CAD tiene una presentación clínica más sintomática y larvada que la hipoglucemia, las que muchas veces suelen resolverse de forma ambulatoria. En pacientes con $\mathrm{CH}$, la mediana de la edad fue 47 años, y la mayoría fue población femenina. En los dos estudios previos, realizados en el mismo nosocomio, se obtuvieron resultados muy similares. ${ }^{4,9}$ Esto puede deberse a que históricamente el HNAL ha sido un nosocomio donde se atendía principalmente a la población femenina $\mathrm{y}$, actualmente, todavía cuenta con más salas de hospitalización de mujeres que de varones. Además, la frecuencia hallada de pacientes con DM1 y DM2 fue similar a la descrita en el estudio de Manrique y col., donde la mayoría fueron pacientes con DM2. ${ }^{4}$ Esto puede explicarse porque en nuestro país la DM1 tiene muy baja prevalencia. Cabe resaltar que casi la mitad de los pacientes con $\mathrm{CH}$ tuvieron diabetes de reciente diagnóstico, similar al estudio mencionado. ${ }^{4}$ Además, en este estudio se observó que el $20 \%$ de los pacientes con $\mathrm{CH}$ tuvieron una $\mathrm{CAD}$ anterior, consistente con otro estudio. ${ }^{6}$ Ambos hallazgos pueden deberse a que en nuestro país existe una falta de cultura de prevención en salud, barreras culturales que desplazan la medicina convencional por la alternativa, falta de conciencia de enfermedad y factores económicos que limitan el acceso a los servicios de salud.
Todo esto hace que estos episodios puedan ocurrir más de una vez.

El factor precipitante más frecuente de las $\mathrm{CH}$ fueron las infecciones, tal y como ha sido descrito en un estudio con población mexicana. ${ }^{10}$ La infección predominante en nuestra serie fue la gastroenterocolitis aguda (GECA), mientras que para dicho estudio fue la infección del tracto urinario (ITU). Esto podría explicarse porque la recolección de datos de los casos se realizó en época de verano, temporada en la cual la GECA es más prevalente. La falta de tratamiento no fue un desencadenante frecuente, al contrario de un estudio con población española, donde el tratamiento irregular fue el precipitante más frecuente. ${ }^{11}$ En cuanto a los pacientes que presentaron hipoglucemia, se observó que todos padecían DM2. Si bien la hipoglucemia ocurre más frecuentemente en pacientes con DM1, como ya se mencionó líneas arriba, en nuestro país hay una mayor prevalencia de DM2. Cabe mencionar que la mayoría de pacientes con hipoglucemia usaba glibenclamida, a diferencia de estudios realizados en España en los que solo el $14,7 \%$ y $4,5 \%$ respectivamente, utilizaban sulfonilureas. ${ }^{11,12}$ Esto probablemente se deba a que en países de primer mundo el uso de estos fármacos es cada vez menos frecuente, mientras que en nuestro país son brindados por el Estado a los hospitales como hipoglucemiantes de primera línea, por motivos económicos. ${ }^{3}$ Adicionalmente al uso de sulfonilureas, la falta de ingesta alimentaria fue el desencadenante más frecuente en pacientes con hipoglucemia.

Dentro de las limitaciones del estudio, cabe recordar que se trata de una serie de casos, por lo que la comparación con otros resultados es únicamente referencial. No obstante, consideramos que las fortalezas de este estudio fueron la actualización de la descripción de las complicaciones agudas de la diabetes en el HNAL y el tipo de la población estudiada. La población de estudio difiere de la mayoría de reportes en la literatura dado que se realizó en pacientes que acudieron al servicio de emergencia, cuyas características pueden variar respecto a los pacientes con DM que son hospitalizados.

\section{REFERENCIAS BIBLIOGRÁFICAS}

I. Rozman C, Cardellach F. Medicina Interna. 19th ed. Barcelona, España: Elsevier España; 2020.

2. International Diabetes Federation. IDF Atlas 9th edition and other resources [Internet]. Bruselas, Bélgica: 2019. [Consultado el II de septiembre de 202I]. URL disponible en: https://www.diabetesatlas. org

3. American Diabetes Association. 2. Classification and diagnosis of diabetes: Standards of Medical Care in Diabetes 2021. Diabetes Care. 202 I; 44 (Supl. I).

4. Manrique H, Ramos M, Medina S, Talaverano O, Pinto V, Solís V. Características epidemiológicas de las crisis hiperglicémicas. Revista Médica Herediana. 20I4; (25):21-25.

5. Ticse R, Alán-Peinado, Baiocchi-Castro L. Características demográficas y epidemiológicas de pacientes con diabetes mellitus tipo 2 hospitalizados por cetoacidosis diabética en un hospital general de Lima-Perú. Revista Médica Herediana. 20।4; (25): 5-I2.

6. Pinto ME, Villena JE, Villena AE. Diabetic ketoacidosis in Peruvian patients with type 2 diabetes mellitus. Endocr Pract. 2008; 14(4): 442-6. 
7. González N, Rodríguez E, Manrique H. Características clínicas y factores asociados a morbilidad intrahospitalaria en los pacientes con diabetes mellitus tipo 2. Rev Soc Peru Med Interna. 20I3; 26(4): 159- 165.

8. Condori Zevallos M. Factores de riesgo asociados a crisis hiperglicémicas en pacientes diabéticos tipo 2 hospitalizados del H.R.H.D. durante 20I3-20I7. Tesis universitaria. Universidad Nacional de San Agustín, Facultad de Medicina; 2018.

9. Manrique H, Calderón J, Soto A, Calle A, Solís J, Castillo O, et al. Cetoacidosis diabética: una complicación frecuente de la diabetes tipo 2 en hispanoamericanos. Revista Médica Herediana. 2014; (25): I4I-I47

10. Domínguez M, Calderón M, Armas R. Características clínicas epidemiológicas de las complicaciones agudas de la diabetes en el servicio de Urgencias del Hospital General de Atizapán. Revista de la Facultad de Medicina de la UNAM. 20I3; (56): 25-36.

II. Sanz Almazan, M; Montero Carretero, T; Sanchez Ramon, S; Jorge Bravo, T; Crespo Soto, C. Estudio descriptivo de las complicaciones agudas diabéticas atendidas en un servicio de urgencias hospitalario. Emergencias: Revista de la Sociedad Española de Medicina de Urgencias y Emergencias. 2017; 4(29): 245-248.

12. Jordi Caballero-Corchuelo*, Fernando Guerrero-Pérez, Paula García-Sancho de la Jordana y Manuel Pérez- Marave. Análisis de las características de los pacientes con diabetes mellitus que consultan por hipoglucemia en el servicio de urgencias de un hospital terciario. Endocrinología, Diabetes y Nutrición. 2019; 66(I): I925.

\section{CORRESPONDENCIA:}

Omar Fabricio Zanoni-Ramos,

omar.zanoni.r@upch.pe

Fecha de recepción: 19-09-2021.

Fecha de aceptación: 30-II-202I.

Financiamiento: por los autores.

Declaración de Conflicto de interés: ninguno, según los autores. 